\title{
A wave equation describing the generation of field-aligned current in the magnetosphere
}

\author{
Masahiro Itonaga $^{1}$, Akimasa Yoshikawa ${ }^{2}$, and Shigeru Fujita ${ }^{3,4}$ \\ ${ }^{1}$ Faculty of Education, Yamaguchi University, Yamaguchi 753-8513, Japan \\ ${ }^{2}$ Department of Earth and Planetary Sciences, Kyushu University, Fukuoka 812-8581, Japan \\ ${ }^{3}$ Meteorological College, Kashiwa 277-0852, Japan \\ ${ }^{4}$ Department of Geophysics, Kyoto University, Kyoto 606-8502, Japan
}

(Received September 20, 1999; Revised July 4, 2000; Accepted July 4, 2000)

\begin{abstract}
A wave equation describing the generation of field-aligned current (FAC) in the magnetosphere is derived. The equation has four source terms. The first and second terms represent the effects of inhomogeneous Alfvén speed $\left(V_{\mathrm{A}}\right)$ and curvilinear magnetic field line, respectively. The perpendicular perturbation inertial current produces the perturbation FAC via these effects. Around the magnetic equator in the region of dipolar magnetic field where $V_{\mathrm{A}}$ is inversely proportional to the power of the radial distance from the Earth's center, the first and second terms have magnitudes of the same order and their signs are identical. The first term dominates over the second one around the region where the gradient of $V_{\mathrm{A}}$ is sharp and vice versa around the position where the stretched field line intersects the magnetic equator. The third and fourth terms are related to the diamagnetic current. When the unperturbed magnetic pressure has an inhomogeneous distribution, the perpendicular diamagnetic current due to the perturbation of the plasma pressure yields the perturbation FAC (third term). When the perpendicular diamagnetic current flows in the unperturbed state, the perturbations of the magnetic and plasma pressures also bring about the perturbation FAC (fourth term). In the case of $\beta \sim 1$, the third and fourth terms have magnitudes of the same order. If the disturbance bears a diamagnetic property, this would be especially the case. However, if the disturbance propagates perpendicularly to the ambient magnetic field, the perturbation FAC would be little generated by the fourth term.
\end{abstract}

\section{Introduction}

The field-aligned current (FAC) is a key element in the magnetosphere-ionosphere coupling and the elucidation of its generation mechanism is one of the most important subjects in magnetospheric physics. In the magnetohydrodynamic (MHD) limit, the FAC is not constrained by dynamical conditions describing motion along the magnetic field. Since the electron parallel mobility is assumed to be infinite, the FAC is simply determined by a current closure requirement that the divergence of current density vanishes everywhere. After most of theoretical works on the FAC (Hasegawa and Sato, 1979; Sato and Iijima, 1979; Sato, 1982; Vasyliunas, 1984), such a requirement also becomes the starting point along with the momentum equation in the present study.

In a warm magnetized plasma there exist three fundamental MHD waves (Dungey, 1968). One is a shear Alfvén wave, and others are fast and slow magnetosonic waves. The FAC is carried only by the shear Alfvén wave. Owing to the effects of inhomogeneous medium and curvilinear magnetic field line, however, these three waves are generally coupled in the magnetosphere. Therefore, the generation of FAC is strongly connected with the generation and propagation of magnetosonic disturbances. Southwood and Saunders (1985) investigated the curvature coupling of slow magne- tosonic and shear Alfvén waves in the magnetotail. Ohtani et al. (1989) derived a system of ordinary differential equations describing the coupling between shear Alfvén and slow magnetosonic waves. They paid attention to the combined effect between pressure gradient and field-line curvature as a coupling mechanism. Southwood and Kivelson (1991) presented an equation describing the time evolution of FAC illustrating the very direct relationship between the transport of FAC and the intermediate (Alfvén) MHD mode. They identified two important categories of FAC's. One is driven by the perpendicular inertial current and the other by the perpendicular pressure gradient. These works treat partially the coupling of FAC (or shear Alfvén wave) with magnetosonic waves. However, it has not been comprehensively studied the way how the effects of inhomogeneous medium and curvilinear magnetic field line produce such a coupling.

In the present paper we will derive a wave equation describing the generation of FAC in the warm magnetized plasma. This is a generalization of the equation given by Itonaga and Yoshikawa (1996) for the cold plasma. The derived wave equation presents a deep insight into the coupling of the FAC with the inertial and diamagnetic currents associated with magnetosonic disturbances via the effects of inhomogeneous medium and curvilinear magnetic field line. 


\section{Wave Equation}

In the present study it is assumed that the magnetosphere is filled with a warm magnetized plasma. In the warm plasma linearized one-fluid equations become

$$
\begin{gathered}
\rho \frac{\partial \delta \mathbf{u}}{\partial t}=-\nabla \delta p+\mathbf{j}_{\perp}^{(\mathrm{d})} \times \delta \mathbf{B}+\delta \mathbf{j}_{\perp} \times \mathbf{B}, \\
\nabla \times \delta \mathbf{E}_{\perp}=-\frac{\partial \delta \mathbf{B}}{\partial t}, \\
\nabla \times \delta \mathbf{B}=\mu_{0} \delta \mathbf{j}, \\
\delta \mathbf{E}_{\perp}+\delta \mathbf{u}_{\perp} \times \mathbf{B}=\mathbf{0},
\end{gathered}
$$

where $\delta \mathbf{u}, \delta \mathbf{E}, \delta \mathbf{B}, \delta \mathbf{j}$ and $\delta p$ are the perturbation flow velocity, electric field, magnetic field, current density and plasma pressure, respectively. In the present paper the perturbation quantity is denoted with the prefix $\delta$. Further, $\rho$ and $\mathbf{B}$ are the unperturbed plasma density and magnetic field, and $\mu_{0}$ is the permeability of vacuum. The subscript $\perp$ stands for a vector component perpendicular to $\mathbf{B}$. It is assumed in Eq. (1) that the flow velocity $\mathbf{u}$ disappears and the perpendicular diamagnetic current $\mathbf{j}_{\perp}^{\text {(d) }}$ flows in the unperturbed state, where $\mathbf{j}_{\perp}^{\text {(d) }}$ is given by

$$
\mathbf{j}_{\perp}^{(\mathrm{d})}=\frac{1}{B^{2}} \mathbf{B} \times \nabla_{\perp} p
$$

with $p$ and $B$ denoting the unperturbed plasma pressure and the magnitude of $\mathbf{B}$, respectively. It is also supposed that the FAC vanishes in the unperturbed state (or $\nabla \cdot \mathbf{j}_{\perp}^{(\mathrm{d})}=0$ ). Then, the ambient magnetic field $\mathbf{B}$ can be represented by Euler potentials (Stern, 1967). The vanishing of $\nabla \cdot \mathbf{j}_{\perp}^{\text {(d) }}$ means that $\nabla_{\perp} p$ is parallel to $\nabla_{\perp} p_{B}$, where $p_{B}=B^{2} / 2 \mu_{0}$ is the unperturbed magnetic pressure.

Taking the vector product between each side of Eq. (1) and B, we have

$$
\delta \mathbf{j}_{\perp}=\delta \mathbf{j}_{\perp}^{(\mathrm{i})}+\delta \mathbf{j}_{\perp}^{(\mathrm{d})}-\frac{1}{2 p_{B}} \delta p_{B} \mathbf{j}_{\perp}^{(\mathrm{d})},
$$

where $\delta p_{B}=B \delta B_{\|} / \mu_{0}$ is the perturbation magnetic pressure. The subscript $\|$ stands for a scalar component parallel to $\mathbf{B}$. Further, $\delta \mathbf{j}_{\perp}^{(\mathrm{i})}$ and $\delta \mathbf{j}_{\perp}^{(\mathrm{d})}$ are the perturbation inertial and diamagnetic currents, which are given by

$$
\begin{aligned}
\delta \mathbf{j}_{\perp}^{(\mathrm{i})} & =\frac{1}{B^{2}} \mathbf{B} \times\left(\rho \frac{\partial \delta \mathbf{u}_{\perp}}{\partial t}\right), \\
\delta \mathbf{j}_{\perp}^{(\mathrm{d})} & =\frac{1}{B^{2}} \mathbf{B} \times \nabla_{\perp} \delta p,
\end{aligned}
$$

flowing perpendicularly to $\mathbf{B}$. The third term in the righthand side of Eq. (6) is also the diamagnetic current due to the perturbation of the magnetic pressure, while $\delta \mathbf{j}_{\perp}^{(\mathrm{d})}$ arises from that of the plasma pressure. From $\nabla \cdot \delta \mathbf{j}=0$ we have

$$
\nabla \cdot \delta \mathbf{j}_{\perp}=-B \frac{\partial}{\partial s}\left(\frac{\delta j_{\|}}{B}\right),
$$

where the variable $s$ is a distance measured along the magnetic field line and increases in the direction of $\mathbf{B}$. Taking the divergence of both sides of Eq. (6), then, we obtain

$$
B \frac{\partial}{\partial s}\left(\frac{\delta j_{\|}}{B}\right)=-\nabla \cdot \delta \mathbf{j}_{\perp}^{(\mathrm{i})}-\nabla \cdot\left(\delta \mathbf{j}_{\perp}^{(\mathrm{d})}-\frac{1}{2 p_{B}} \delta p_{B} \mathbf{j}_{\perp}^{(\mathrm{d})}\right) .
$$

Using $\nabla \times \mathbf{B}=\mu_{0} \mathbf{j}_{\perp}^{(\mathrm{d})}$, we acquire

$$
\nabla \cdot \delta \mathbf{j}_{\perp}^{(\mathrm{d})}=\frac{1}{2 p_{B}} \nabla_{\perp} \delta p \cdot \mathbf{j}_{\perp}^{(\mathrm{d})}-\frac{1}{p_{B}} \nabla_{\perp} p_{B} \cdot \delta \mathbf{j}_{\perp}^{(\mathrm{d})}
$$

from the divergence of both sides of Eq. (8). Further, noting that $\nabla_{\perp} p_{B}$ is parallel to $\nabla_{\perp} p$, we have

$$
\nabla \cdot\left(\frac{1}{2 p_{B}} \delta p_{B} \mathbf{j}_{\perp}^{(\mathrm{d})}\right)=\frac{1}{2 p_{B}} \nabla_{\perp} \delta p_{B} \cdot \mathbf{j}_{\perp}^{(\mathrm{d})} .
$$

From these equations the divergence of the perpendicular perturbation diamagnetic current, $\delta \mathbf{j}_{\perp}^{\text {(d) }}-\delta p_{B} \mathbf{j}_{\perp}^{(\mathrm{d})} / 2 p_{B}$, becomes

$$
\begin{aligned}
\nabla & \cdot\left(\delta \mathbf{j}_{\perp}^{(\mathrm{d})}-\frac{1}{2 p_{B}} \delta p_{B} \mathbf{j}_{\perp}^{(\mathrm{d})}\right) \\
& =-\frac{1}{p_{B}} \nabla_{\perp} p_{B} \cdot \delta \mathbf{j}_{\perp}^{(\mathrm{d})}-\frac{1}{2 p_{B}} \nabla_{\perp}\left(\delta p_{B}-\delta p\right) \cdot \mathbf{j}_{\perp}^{(\mathrm{d})} \cdot(10)
\end{aligned}
$$

In order to derive a wave equation describing the generation of FAC in the magnetosphere, we consider the time derivative of parallel component of Eq. (3), that is,

$$
\frac{\partial \delta j_{\|}}{\partial t}=\frac{1}{\mu_{0}}\left[-\frac{\partial}{\partial s}\left(\nabla \cdot \delta \mathbf{E}_{\perp}\right)+\left(\nabla^{2} \delta \mathbf{E}_{\perp}\right)_{\|}\right],
$$

where Eq. (2) has been used to eliminate $\partial \delta \mathbf{B} / \partial t$. Further, taking the time derivative of Eq. (4) and using Eq. (7), we obtain

$$
\frac{\partial \delta \mathbf{E}_{\perp}}{\partial t}=\mu_{0} V_{\mathrm{A}}^{2} \delta \mathbf{j}_{\perp}^{(\mathrm{i})},
$$

where $V_{\mathrm{A}}=B / \sqrt{\mu_{0} \rho}$ is the Alfvén speed. Then, the time derivative of Eq. (11) along with Eq. (12) results in

$$
\frac{\partial^{2} \delta j_{\|}}{\partial t^{2}}=-\frac{\partial}{\partial s}\left[\nabla \cdot\left(V_{\mathrm{A}}^{2} \delta \mathbf{j}_{\perp}^{(\mathrm{i})}\right)\right]+\left[\nabla^{2}\left(V_{\mathrm{A}}^{2} \delta \mathbf{j}_{\perp}^{(\mathrm{i})}\right)\right]_{\|} .
$$

Using Eqs. (9) and (10), we have

$$
\begin{aligned}
\nabla \cdot\left(V_{\mathrm{A}}^{2} \delta \mathbf{j}_{\perp}^{(\mathrm{i})}\right)= & V_{\mathrm{A}}^{2} \nabla \cdot \delta \mathbf{j}_{\perp}^{(\mathrm{i})}+\nabla_{\perp} V_{\mathrm{A}}^{2} \cdot \delta \mathbf{j}_{\perp}^{(\mathrm{i})} \\
= & -V_{\mathrm{A}}^{2} B \frac{\partial}{\partial s}\left(\frac{\delta j_{\|}}{B}\right)+\frac{V_{\mathrm{A}}^{2}}{p_{B}} \nabla_{\perp} p_{B} \cdot \delta \mathbf{j}_{\perp}^{(\mathrm{d})} \\
& +\frac{V_{\mathrm{A}}^{2}}{2 p_{B}} \nabla_{\perp}\left(\delta p_{B}-\delta p\right) \cdot \mathbf{j}_{\perp}^{(\mathrm{d})} \\
& +\nabla_{\perp} V_{\mathrm{A}}^{2} \cdot \delta \mathbf{j}_{\perp}^{(\mathrm{i})} .
\end{aligned}
$$

The combination of the above equation and Eq. (13) gives rise to

$$
\begin{aligned}
\frac{1}{V_{\mathrm{A}}^{2}} \frac{\partial^{2} \delta j_{\|}}{\partial t^{2}}- & \frac{1}{V_{\mathrm{A}}^{2}} \frac{\partial}{\partial s}\left[V_{\mathrm{A}}^{2} B \frac{\partial}{\partial s}\left(\frac{\delta j_{\|}}{B}\right)\right] \\
= & -\frac{1}{V_{\mathrm{A}}^{2}} \frac{\partial}{\partial s}\left(\nabla_{\perp} V_{\mathrm{A}}^{2} \cdot \delta \mathbf{j}_{\perp}^{(\mathrm{i})}\right) \\
& +\frac{1}{V_{\mathrm{A}}^{2}}\left[\nabla^{2}\left(V_{\mathrm{A}}^{2} \delta \mathbf{j}_{\perp}^{(\mathrm{i})}\right)\right]_{\|} \\
& -\frac{1}{V_{\mathrm{A}}^{2}} \frac{\partial}{\partial s}\left(\frac{V_{\mathrm{A}}^{2}}{p_{B}} \nabla_{\perp} p_{B} \cdot \delta \mathbf{j}_{\perp}^{(\mathrm{d})}\right) \\
& -\frac{1}{V_{\mathrm{A}}^{2}} \frac{\partial}{\partial s}\left[\frac{V_{\mathrm{A}}^{2}}{2 p_{B}} \nabla_{\perp}\left(\delta p_{B}-\delta p\right) \cdot \mathbf{j}_{\perp}^{(\mathrm{d})}\right] .(1
\end{aligned}
$$




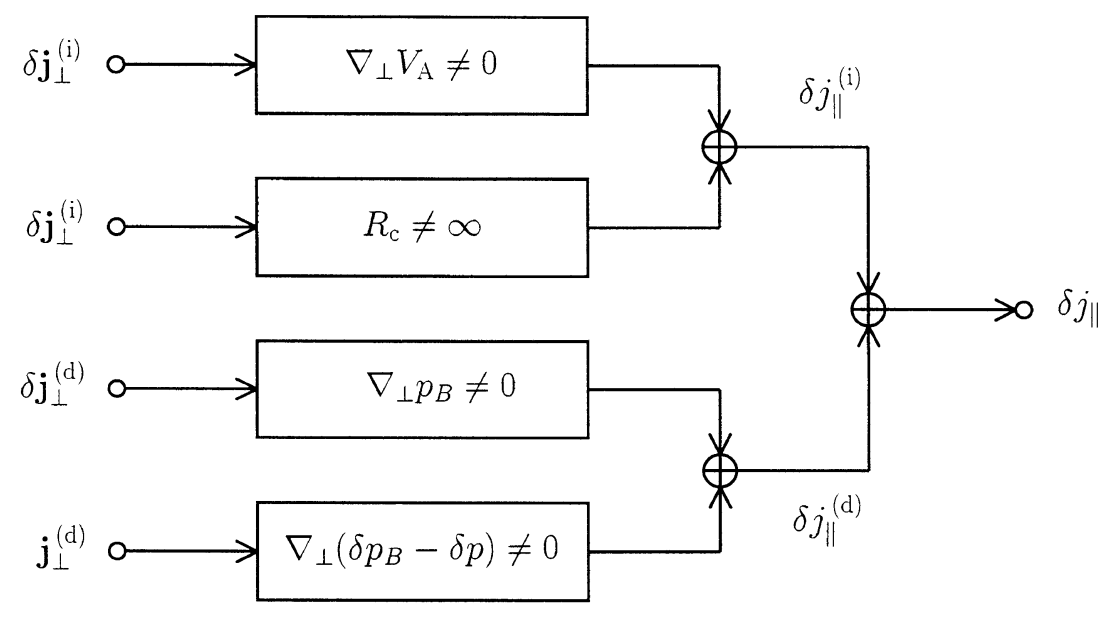

Fig. 1. Schematic diagram for the generation of perturbation FAC. When the Alfvén speed is inhomogeneous $\left(\nabla_{\perp} V_{\mathrm{A}} \neq 0\right)$ and the magnetic field line is curvilinear $\left(R_{\mathrm{c}} \neq \infty\right)$, the perpendicular perturbation inertial current $\delta \mathbf{j}_{\perp}^{(\mathrm{i})}$ produces the perturbation $\mathrm{FAC} \delta j_{\|}{ }_{\|}^{(\mathrm{i})}$. When the perturbation magnetic pressure is inhomogeneous $\left(\nabla_{\perp} p_{B} \neq 0\right)$, the perpendicular perturbation diamagnetic current $\delta \mathbf{j}_{\perp}^{(\mathrm{d})}$ yields the perturbation FAC $\delta j_{\|}{ }^{(\mathrm{d})}$. When the perpendicular diamagnetic current $\mathbf{j}_{\perp}^{(\mathrm{d})}$ flows in the unperturbed state, the perturbations of the magnetic and plasma pressures $\left(\nabla_{\perp}\left(\delta p_{B}-\delta p\right) \neq 0\right)$ also bring about the perturbation $\operatorname{FAC} \delta j_{\|}^{(\mathrm{d})}$. The perturbation FAC $\delta j_{\|}$consists of $\delta j_{\|}^{(\mathrm{i})}$ and $\delta j_{\|}^{(\mathrm{d})}$ due to the perpendicular perturbation inertial and diamagnetic currents.

This is the desired wave equation for the perturbation FAC $\delta j_{\|}$with four source terms and describes an aspect that $\delta j_{\|}$ is successively generated owing to the source terms. Here, it should be noted that Eq. (14) has been never derived to solve the coupling problem of MHD waves self-consistently. When we have some knowledges of the distribution of $\delta \mathbf{j}_{\perp}^{(\mathrm{i})}$ and/or those of $\delta p$ and $\delta p_{B}$, we can say something about the generation of FAC using Eq. (14). The usage of Eq. (14) is similar to that of the expression put forward by Hasegawa and Sato (1979). In the cold plasma, because of $\mathbf{j}_{\perp}^{\text {(d) }}=$ $\delta \mathbf{j}_{\perp}^{(\mathrm{d})}=\mathbf{0}$, Eq. (14) leads to the equation given by Itonaga and Yoshikawa (1996).

\section{Discussion}

The first and second terms in the righthand side of Eq. (14) indicate the effects of inhomogeneous Alfvén speed and curvilinear magnetic field line, respectively. The second term vanishes in the case of straight field line or $R_{\mathrm{c}}=\infty$, where $R_{\mathrm{c}}$ denotes the radius of curvature of the magnetic field line. When there exist the inhomogeneity of the Alfvén speed and the curvature of the magnetic field line, the perturbation FAC $\delta j_{\|}$is produced by the perpendicular perturbation inertial current $\delta \mathbf{j}_{\perp}^{(\mathrm{i})}$. On the other hand, the third term shows that $\delta j_{\|}$is caused by the perpendicular perturbation diamagnetic current $\delta \mathbf{j}_{\perp}^{(\mathrm{d})}$ in case of the existence of inhomogeneity of the unperturbed magnetic pressure. The generation of FAC via the third term was investigated by Southwood and Kivelson (1991) in a quite different form. Further, when the perpendicular diamagnetic current flows in the unperturbed state, the perturbations of the magnetic and plasma pressures bring about $\delta j_{\|}$, as seen from the fourth term. Figure 1 is a schematic diagram for the generation of perturbation FAC.

The cold plasma approximation is suitable for the nearEarth region such as the plasmasphere. In such a region the inertial current plays an essential role in the generation of FAC. Itonaga and Yoshikawa (1996) considered the inertial current in case of the dipolar magnetic field. In the present study, introducing Euler potentials, $\Phi$ and $\phi$ (Stern, 1967; Southwood and Kivelson, 1991), we represent the ambient magnetic field $\mathbf{B}$ with

$$
\mathbf{B}=\nabla \Phi \times \nabla \phi .
$$

We can require $\nabla \Phi \cdot \nabla \phi=0$ without loss of generality. The Euler potentials $\Phi$ and $\phi$ are constant along each magnetic field line and label the meridional and longitudinal directions, respectively. Further, we introduce an orthogonal coordinate system $(\Phi, \phi, \chi)$ based on the Euler potentials, where $\chi$ varies along each magnetic field line and increases in the direction of $\mathbf{B}$. Figure 2 illustrates the orthogonal coordinate system. The scale factors of $\Phi, \phi$ and $\chi$ are denoted by $h_{\Phi}$, $h_{\phi}$ and $h_{\chi}$, respectively.

In the following it is assumed that the characteristic length in spatial variation of the perturbation quantity is much smaller than those of the scale factors and the Alfvén speed.

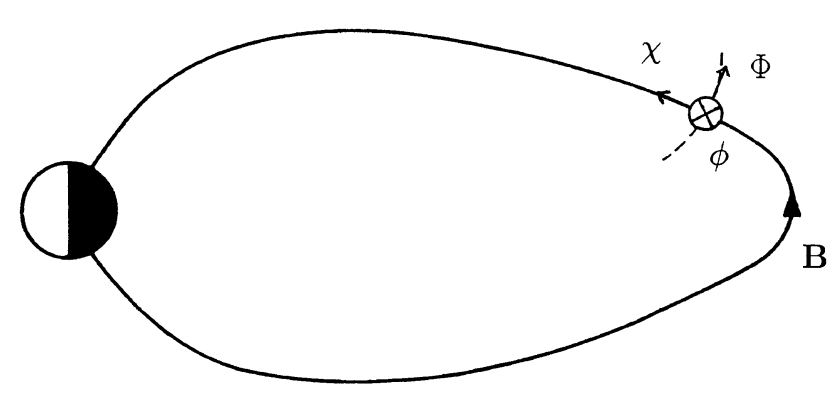

Fig. 2. Orthogonal coordinate system $(\Phi, \phi, \chi)$. The Euler potentials $\Phi$ and $\phi$, which are constant along each magnetic field line, label the meridional and longitudinal directions, repectively, while $\chi$ varies along each field line and increases in the direction of the ambient magnetic field B. 


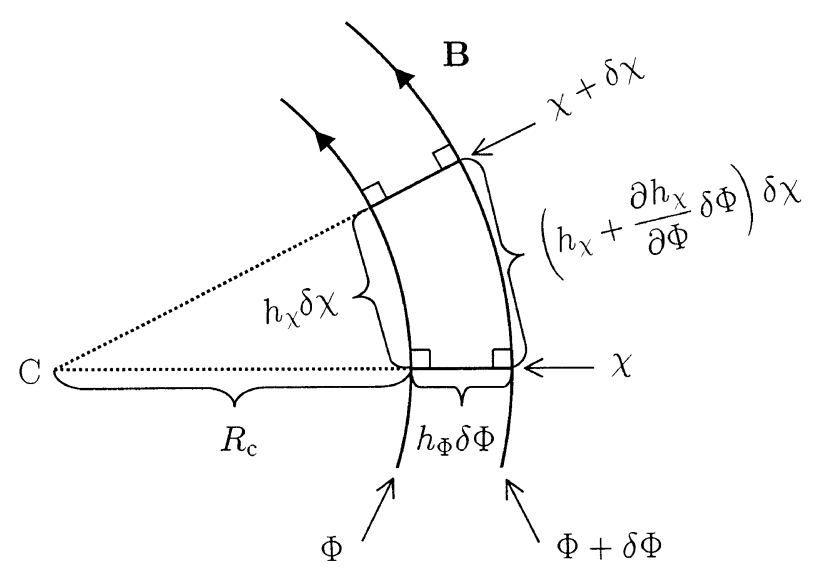

Fig. 3. Adjacent magnetic field lines designated by $\Phi$ and $\Phi+\delta \Phi$ in a plane. The point $\mathrm{C}$ is the center and $R_{\mathrm{c}}$ is the radius of curvature of the field line designated by $\Phi$ at the position $\chi$. If $\delta \chi$ and $\delta \Phi$ are fully small, the length of the portion between $\chi$ and $\chi+\delta \chi$ is $h_{\chi} \delta \chi$ for the field line designated by $\Phi$ and $\left(h_{\chi}+\delta \Phi \partial h_{\chi} / \partial \Phi\right) \delta \chi$ for that by $\Phi+\delta \Phi$.

Then, we can obtain

$$
\begin{aligned}
& \frac{1}{V_{\mathrm{A}}^{2}}\left[\nabla^{2}\left(V_{\mathrm{A}}^{2} \delta \mathbf{j}_{\perp}^{(\mathrm{i})}\right)\right]_{\|} \\
& \simeq-2\left(\frac{1}{h_{\Phi}} \frac{1}{h_{\chi}} \frac{\partial h_{\Phi}}{\partial \chi} \frac{1}{h_{\Phi}} \frac{\partial \delta j_{\Phi}^{(\mathrm{i})}}{\partial \Phi}\right. \\
&\left.-\frac{1}{h_{\chi}} \frac{1}{h_{\Phi}} \frac{\partial h_{\chi}}{\partial \Phi} \frac{1}{h_{\chi}} \frac{\partial \delta j_{\Phi}^{(\mathrm{i})}}{\partial \chi}\right) \\
&-2\left(\frac{1}{h_{\phi}} \frac{1}{h_{\chi}} \frac{\partial h_{\phi}}{\partial \chi} \frac{1}{h_{\phi}} \frac{\partial \delta j_{\phi}^{(\mathrm{i})}}{\partial \phi}\right. \\
&\left.-\frac{1}{h_{\chi}} \frac{1}{h_{\phi}} \frac{\partial h_{\chi}}{\partial \phi} \frac{1}{h_{\chi}} \frac{\partial \delta j_{\phi}^{(\mathrm{i})}}{\partial \chi}\right) .
\end{aligned}
$$

If the scale factors are symmetric about the magnetic equator $(\chi=0)$ and their $\phi$-dependences are negligibly small, the above equation leads to

$$
\frac{1}{V_{\mathrm{A}}^{2}}\left[\nabla^{2}\left(V_{\mathrm{A}}^{2} \delta \mathbf{j}_{\perp}^{(\mathrm{i})}\right)\right]_{\|} \simeq 2 \frac{1}{h_{\chi}} \frac{1}{h_{\Phi}} \frac{\partial h_{\chi}}{\partial \Phi} \frac{1}{h_{\chi}} \frac{\partial \delta j_{\Phi}^{(\mathrm{i})}}{\partial \chi}
$$

around the magnetic equator. Further, assuming that the $\phi-$ dependence of $V_{\mathrm{A}}$ is also negligibly small, we have

$$
\begin{aligned}
& -\frac{1}{V_{\mathrm{A}}^{2}} \frac{\partial}{\partial s}\left(\nabla_{\perp} V_{\mathrm{A}}^{2} \cdot \delta \mathbf{j}_{\perp}^{(\mathrm{i})}\right) \\
& \simeq-\frac{1}{V_{\mathrm{A}}^{2}} \frac{1}{h_{\Phi}} \frac{\partial V_{\mathrm{A}}^{2}}{\partial \Phi} \frac{1}{h_{\chi}} \frac{\partial \delta j_{\Phi}^{(\mathrm{i})}}{\partial \chi} .
\end{aligned}
$$

Around the magnetic equator in most regions of the magnetosphere, $V_{\mathrm{A}}$ decreases as the position goes away from the Earth (or $\Phi$ increases), that is, $\partial V_{\mathrm{A}}^{2} / \partial \Phi<0$. On the other hand, $\partial h_{\chi} / \partial \Phi$ has generally a positive value. As seen from Eqs. (15) and (16), thus, the first and second terms in the righthand side of Eq. (14) have the same sign. Assuming that the meridian (or the surface at which $\phi$ is constant) is a plane, we consider two magnetic field lines designated by $\Phi$ and $\Phi+\delta \Phi$ lying in a meridian (Fig. 3). If $\delta \chi$ and $\delta \Phi$ are fully small, the length of the portion between $\chi$ and $\chi+\delta \chi$ is $h_{\chi} \delta \chi$ for the magnetic field line designated by $\Phi$ and $\left(h_{\chi}+\delta \Phi \partial h_{\chi} / \partial \Phi\right) \delta \chi$ for that by $\Phi+\delta \Phi$. Then, from

$$
\frac{h_{\chi} \delta \chi}{R_{\mathrm{c}}}=\frac{\left(h_{\chi}+\delta \Phi \partial h_{\chi} / \partial \Phi\right) \delta \chi}{R_{\mathrm{c}}+h_{\Phi} \delta \Phi}
$$

where $R_{\mathrm{c}}$ denotes the radius of curvature of the magnetic field line designated by $\Phi$ (Fig. 3), we have

$$
\frac{1}{h_{\chi}} \frac{1}{h_{\Phi}} \frac{\partial h_{\chi}}{\partial \Phi}=\frac{1}{R_{\mathrm{c}}} \text {. }
$$

In the region of dipolar magnetic field, $R_{\mathrm{c}} \sim r$, where $r$ is the radial distance of the position from the Earth's center. Then, if $V_{\mathrm{A}}$ is inversely proportional to the power of $r$ around the magnetic equator, the first and second terms will have magnitudes of the same order there.

Around the position where the stretched magnetic field line intersects the magnetic equator, as in the magnetotail, $R_{\mathrm{c}}$ is very small and so the second term in the righthand side of Eq. (14) will dominate over the first one. Southwood and Saunders (1985) investigated the curvature coupling of slow magnetosonic and shear Alfvén waves in the magnetotail. Ohtani et al. (1989) also found that the combined effect between the plasma pressure gradient and the field line curvature can be a free energy source of ballooninginterchange instability for exciting the coupled oscillation of shear Alfvén and slow magnetosonic modes. The diamagnetic current associated with the slow magnetosonic wave would certainly play an important role in the generation of FAC (or shear Alfvén wave) in the magnetotail. As seen from Eq. (14), however, it is not the diamagnetic current but the inertial current that produces the FAC via the curvature effect of magnetic field line. Just inside the plasmapause, on the other hand, $V_{\mathrm{A}}$ has a sharp positive gradient and so the first term in the righthand side of Eq. (14) will be predominant over the second one. The gradient of $V_{\mathrm{A}}$ in the $\Phi$ direction has opposite signs just inside the plasmapause and in its surrounding regions. This might cause a $180^{\circ}$ phase shift in the $H$ component of Pi 2 pulsations around the plasmapause (Itonaga and Yoshikawa, 1996).

In the region where the pressure gradient plays an important role in the plasma motion, the third and fourth terms in the righthand side of Eq. (14) might become comparable to the first and second ones in magnitude, as seen from

$$
\left|\frac{\text { third term }}{\text { first term }}\right| \sim \frac{\left|\nabla_{\perp} \delta p\right|}{\left|\rho \partial \delta \mathbf{u}_{\perp} / \partial t\right|} .
$$

In the case of $\beta \sim 1$ where $\beta=p / p_{B}$, the third and fourth terms have magnitudes of the same order, because

$$
\left|\frac{\text { third term }}{\text { fourth term }}\right| \sim \frac{2}{\beta}\left|\frac{\delta p}{\delta p_{B}-\delta p}\right| .
$$

In a uniform plasma the relation

$$
\delta p_{B}=\left(\frac{V_{\mathrm{A}}}{V_{\mathrm{s}}}\right)^{2} \delta p
$$

holds for the fast magnetosonic wave propagating perpendicularly to $\mathbf{B}$, where $V_{\mathrm{s}}$ is the sound speed. If $V_{\mathrm{A}} \sim V_{\mathrm{S}}$ (or 
$\beta \sim 1), \delta p_{B}$ and $\delta p$ are in phase and have magnitudes of the same order. Also for the real magnetosphere, when the disturbance propagates perpendicularly to $\mathbf{B}$ in the region of $\beta \sim 1$, this might be the case. Then, the perturbation FAC would be little generated by the fourth term. On the other hand, when the disturbance has a diamagnetic property that the phase difference between $\delta p_{B}$ and $\delta p$ is nearly $180^{\circ}$, the fourth term would play an important role in the generation of perturbation FAC as well as the third one.

\section{Concluding Remarks}

In general, there exist plasma convections or flows in the magnetosphere. Then, it might be inappropriate to assume that the flow velocity $\mathbf{u}$ disappears in the unperturbed state. If so, the perpendicular inertial current

$$
\mathbf{j}_{\perp}^{(\mathrm{i})}=\frac{1}{B^{2}} \mathbf{B} \times[\rho(\mathbf{u} \cdot \nabla) \mathbf{u}]
$$

flows and its divergence will give rise to the unperturbed FAC. However, it can be easily shown that Eqs. (1) and (4) also hold under the condition of $u / \sqrt{V_{\mathrm{A}}^{2}+V_{\mathrm{s}}^{2}} \ll 1$, where $u$ is the magnitude of $\mathbf{u}$, even if $\mathbf{u}$ does not get zero in the unperturbed state. This means that Eq. (14) is effective even if the unperturbed FAC due to the divergence of $\mathbf{j}_{\perp}^{(i)}$ flows. Since the convective speed due to the Earth rotation is only $3 \mathrm{~km} / \mathrm{s}$ or so even at $L=6$, the above condition will be sure to hold in the region of dipolar magnetic field relatively near the Earth. In the distant magnetosphere, however, it is thought that the flow speed is much larger than the convective speed. Then, the validity of the condition must be carefully examined in such a region. This is a future subject.

Equation (14) is a linear formula and so it cannot be applied to such a highly nonlinear process as the formation of substorm current wedge with a time scale of about 30 to $60 \mathrm{~min}$ in the magnetosphere. The nonlinear process is nowadays studied directly with numerical simulations (e.g., Tanaka, 1995). It is thought that some small-amplitude quasi-oscillatory currents with time scales of about 1 to 2 min superposed on the large-amplitude slowly-varying wedge current cause Pi 2 pulsations (Lester et al., 1983; Itonaga and Yumoto, 1998). The cross-tail portion of the quasi-oscillatory current wedge generates a fast magnetosonic wave. During its passage within the magnetosphere the magnetosonic wave excites shear Alfvén waves owing to the effects of inhomogeneous medium and curvilinear magnetic field line. Since the formation of quasi-oscillatory wedge current and the associated process have not been yet simulated numerically, the use of
Eq. (14) will be of great advantage to their theoretical studies. In the present study the magnitude of each source term is roughly estimated. However, more precise estimations based on some magnetic field models, such as the Tsyganenko models, and satellite observations are indispensable for the detailed discussion of FAC generation. This is also an important future subject.

Acknowledgments. The authors would like to express their thanks to A. Miura and K. Yumoto for their helpful discussions. This work was partly supported by the Grant-in-Aid for Scientific Research (C), No. 09640532, from The Ministry of Education, Science and Culture. The authors are also very much grateful to two referees who have contributed to the improvement of this paper.

\section{References}

Dungey, J. W., Hydromagnetic waves, in Physics of Geomagnetic Phenomena, edited by S. Matsushita and W. H. Campbell, pp. 913-934, Academic Press, New York, 1968.

Hasegawa, A. and T. Sato, Generation of field aligned current during substorm, in Dynamics of the Magnetosphere, edited by S.-I. Akasofu, pp. 529-542, D. Reidel, Hingham, Mass., 1979.

Itonaga, M. and A. Yoshikawa, The excitation of shear Alfvén wave and the associated modulation of compressional wave in the inner magnetosphere, J. Geomag. Geoelectr., 48, 1451-1459, 1996.

Itonaga, M. and K. Yumoto, ULF waves and the ground magnetic field, $J$. Geophys. Res., 103, 9285-9291, 1998.

Lester, M., W. J. Hughes, and H. Jinger, Polarization pattern of Pi 2 magnetic pulsations and the substorm current wedge, J. Geophys. Res., 88, 79587966, 1983.

Ohtani, S., A. Miura, and T. Tamao, Coupling between Alfvén and slow magnetosonic waves in an inhomogeneous finite- $\beta$ plasma-I. Coupled equations and physical mechanism, Planet. Space Sci., 37, 567-577, 1989

Sato, T., Auroral physics, in Magnetospheric Plasma Physics, edited by A Nishida, pp. 197-243, D. Reidel, Hingham, Mass., 1982.

Sato, T. and T. Iijima, Primary sources of large-scale Birkeland currents, Space Sci. Rev., 24, 347-366, 1979.

Southwood, D. J. and M. G. Kivelson, An approximate description of fieldaligned currents in a planetary magnetic field, J. Geophys. Res., 96, 67-75, 1991

Southwood, D. J. and M. A. Saunders, Curvature coupling of slow and Alfvén MHD waves in a magnetotail field configuration, Planet. Space Sci., 33, 127-134, 1985.

Stern, D., Geomagnetic Euler potentials, J. Geophys. Res., 72, 3995-4005, 1967.

Tanaka, T., Generation mechanisms for magnetosphere-ionosphere current systems deduced from a three-dimensional MHD simulation of the solar wind-magnetosphere-ionosphere coupling processes, J. Geophys. Res., 100, 12,057-12,074, 1995.

Vasyliunas, V. M., Fundamentals of current description, in Magnetospheric Currents, edited by T. A. Potemra, pp. 63-66, AGU, Washington, D.C., 1984

M. Itonaga (e-mail: itonaga@po.cc.yamaguchi-u.ac.jp), A. Yoshikawa (e-mail: yoshi@geo.kyushu-u.ac.jp),andS.Fujita(e-mail: sfujita@typhoon. mc-jma.ac.jp) 